\title{
Electrically tunable Dicke effect in a double-ring resonator
}

\author{
A. E. Çetin* \\ Photonics Center and School of Electrical and Computer Engineering, Boston University, Boston, Massachusetts 02215, USA
}

Ö. E. Müstecaplıŏglu

Department of Physics, Koç University, Sarlyer, Istanbul 34450, Turkey and

Institute of Quantum Electronics, ETH Zurich, Zurich 8093, Switzerland

(Received 12 December 2009; published 13 April 2010)

\begin{abstract}
We study the finite-element method analysis of the Dicke effect using numerical simulations in an all-optical system of an optical waveguide side-coupled to two interacting ring resonators in a liquid crystal environment. The system is shown to exhibit all the signatures of the Dicke effect under active and reversible control by an applied voltage.
\end{abstract}

DOI: 10.1103/PhysRevA.81.043812

PACS number(s): 42.79.Gn, 42.60.Da, 42.50.Nn

\section{INTRODUCTION}

The Dicke effect, substantial narrowing of spectral line shapes due to collisions of radiating and nonradiating atoms, was first described by R. H. Dicke in [1]. The overall line shape consists of a superposition of broad and narrow peaks centered at the transition frequency. This splitting of atomic decay into pairs of fast- and slow-decay channels is closely related to the superradiance phenomenon predicted shortly after description of the Dicke effect [2]. Superradiance is a cooperative spontaneous emission of radiation from an initially excited coherent ensemble of atoms. The slow- and fast-decay channels are respectively called subradiant and superradiant decays of the system. Collective symmetric or antisymmetric states of an ensemble of atoms are in superradiant and subradiant phases, respectively.

In addition to atomic ensembles, the Dicke effect has been extensively studied in other systems, such as photonic crystals [3], plasmonic lattices [4], electronic mesoscopic systems [5-12], and electron waveguides [13,14]. Not all the characteristics of the Dicke effect can be found in these systems. Furthermore, control of the Dicke effect is challenging. Our purpose is to examine the Dicke effect in an all-optical device with active and reversible control. We find that a pair of microring resonators coupled to a waveguide can exhibit all the key signatures of the Dicke effect in a controllable way in a nematic liquid crystal (NLC). Tunable lifetimes of quasibound states in the resonators can be translated into reversible active control of optical signals in multiple-ringresonator configurations. These systems are used for optical communication and signal processing applications such as all-optical logic gates [15] and all-optical memory elements [16].

The system allows for controlled investigations of quantum interference and decoherence. It provides an all-optical analog of the Anderson-Fano model that is a prototypical description of interaction between (quasi)bound and (quasi)continuum states $[17,18]$. By extension of the system to a multiple-ring configuration, quantum phase transitions in the context of

\footnotetext{
*acetin@bu.edu

†omustecap@ku.edu.tr
}

superradiance can be systematically examined and probed (for a review, see [5] and references therein).

This paper is organized as follows. In Sec. II, we explain the theory behind the tuning of the refractive index of the NLC cladding by application of a voltage from electrodes positioned to the right and left sides of the double-ring system. In Sec. III, we analyze the resonance characteristics of the double-ring system without an applied potential, that is, the dependence of the resonance width and tuning on the gap between the two microring resonators. In Sec. IV, we show how we control the resonance width and tuning by applying a potential from electrodes. In Sec. V, we show the methods used in our numerical computations. We reserve Sec. VI for concluding remarks.

\section{TUNING OF THE REFRACTIVE INDEX OF THE NEMATIC LIQUID CRYSTAL CLADDING WITH APPLIED VOLTAGE}

A NLC can be used to tune the resonances of a singlering resonator coupled to a waveguide [19]. To control the linewidths, we introduce another ring to the system as shown in Fig. 1: Two identical microring resonators at a distance $d$ apart are side-coupled to a waveguide. A TE-polarized Gaussian beam (in which the electric field is perpendicular to the plane of the resonators) is sent from the input port of the waveguide. A voltage is provided by two electrodes to change the orientation of the NLC molecules used to clad the resonators. A similar setup without a NLC has been examined for its reflective properties [20,21]. The NLC allows for controllable coupling coefficients between the resonators and the waveguide.

For a single ring, the resonance wavelength is determined by the Fabry-Pérot étalon resonance condition $m \lambda_{m}=2 \pi R n_{\mathrm{eff}}$, where $m=1,2,3, \ldots, \lambda_{m}$ is the wavelength of the $m$ th resonator mode, $R$ is the radius of the microring resonator, and $n_{\text {eff }}$ is the effective refractive index for the waveguide mode $[19,22]$. Proximity coupling by the evanescent tails makes $n_{\text {eff }}$ dependent on the refractive index of the NLC cladding, $n_{\text {clad }}$, which is determined by

$$
\frac{1}{n_{\text {clad }}^{2}}=\frac{\cos ^{2}(\theta)}{n_{e}^{2}}+\frac{\sin ^{2}(\theta)}{n_{o}^{2}},
$$




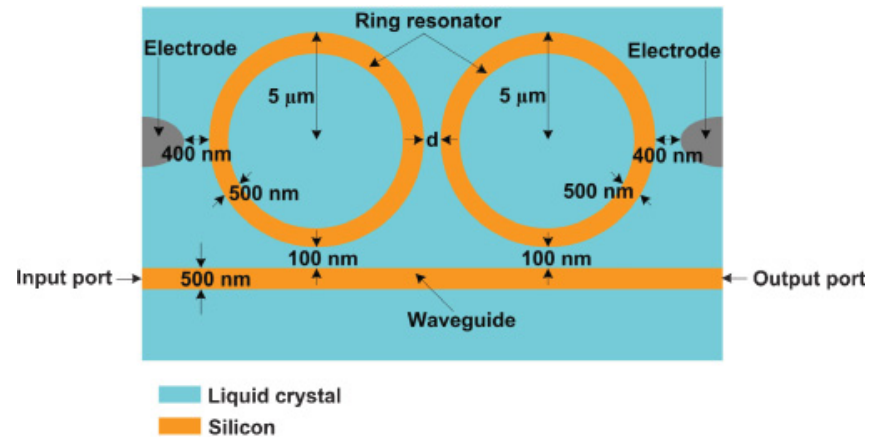

FIG. 1. (Color online) Dimensions of the system consisting of two ring resonators on top of a waveguide fed by two electrodes.

where $n_{e}=1.744$ and $n_{o}=1.517$ at $589 \mathrm{~nm}$ [19], and $\theta$ is the angle of the NLC directors (local pseudovectors in the mean molecular long-axis direction) relative to the radial axis from the origin in the middle of the electrodes $[19,23]$.

When there is no applied field, the NLC is in the isotropic phase so that $n_{\text {clad }}=1.596$ with the assumption that the optical field is too weak to induce any reorientation of the NLC directors (optical Fréedericksz effect). When a sufficiently strong potential is applied from the electrodes, the directors are deformed as shown in Fig. 2. For given elastic properties, $\theta$ is locally determined by the Euler-Lagrange equations to minimize the free-energy density of the NLC. The directors are fully polarized in the applied electric field direction for a potential field much stronger than the elastic contribution [24]. This permits local modulation of $n_{\text {eff }}$ analogous to the electro-optic effect. The electric field lines in Fig. 2, which are numerically determined by the Poisson equation, indicate the director alignments, which are controlled by the boundary conditions at the electrodes [19]. After the $\theta$ distribution is found, the spatially inhomogeneous refractive index of the NLC cladding is calculated by Eq. (1). This is used to propagate the Gaussian beam in the waveguide and to evaluate its transmission. We repeat this process for different potential values and different separations between the rings.

The reflection characteristics of coupled-ring resonators have been examined $[20,21]$ by the transfer matrix formalism $[25,26]$. We follow a computational approach based upon the

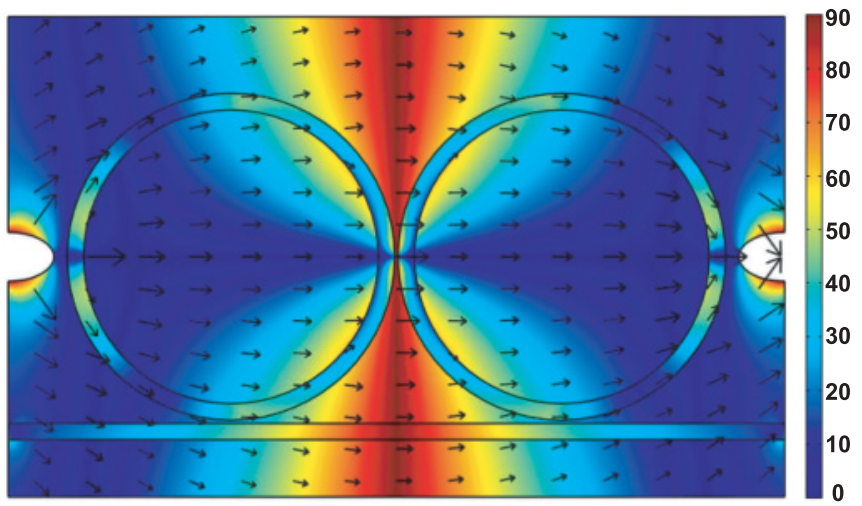

FIG. 2. (Color online) Angular difference (degrees) between the directors of the NLC molecules and the radial axis. The arrows show the direction of the electric field generated by the electrodes at $5 \mathrm{~V}$. finite-element method (FEM) to examine the coupling between the electromagnetic modes as realistically as possible and to treat NLC cladding correctly [27]. We verify that our numerical analysis yields line-shape structures similar to those obtained by the transfer matrix method. We calculate the resonances by evaluating the intensity of the wave at the output port of the waveguide, $I_{\text {out }}$. The spatially inhomogeneous $n_{\text {clad }}$ is used in the coupled wave equations to solve for the modes in the resonator and the waveguide and the evanescent waves in the NLC. Different computational grids are used for each geometry arising when the ring separation is varied. The numerical analysis used to calculate the effect of the applied voltage from the electrodes on tuning of the refractive index of the NLC cladding is explained in Sec. V.

\section{DOUBLE-RING RESONATOR SYSTEM WITHOUT APPLIED VOLTAGE}

The resonances of the system for different $d$ values (the gap distance between the rings) without an applied potential are shown in Fig. 3. A characteristic splitting of the single-ring resonance into four peaks is observed. The effect can be viewed as two separate resonances of the double-ring system, each split into two peaks. However, the pair of resonances of the double-ring system arises when there is some proximity coupling between the rings. The resonance of a single isolated ring splits into two peaks when another ring is placed so as to interact with it. When there are two identical rings, this perturbation is a level repulsion or anticrossing effect. When a waveguide is introduced to the system, further splitting of the resonances occur that results in further peaks. Our explanation is based upon the transfer matrix analysis of this system in Refs. [20] and [21]. There, the authors found numerically that the resonance of an isolated ring splits into two modes, symmetric and antisymmetric, depending on the ring-ring coupling. Further splitting of these modes is due to the coupling of the waveguide. In this work, the NLC environment serves as a means to control the relative strengths of the ring-waveguide
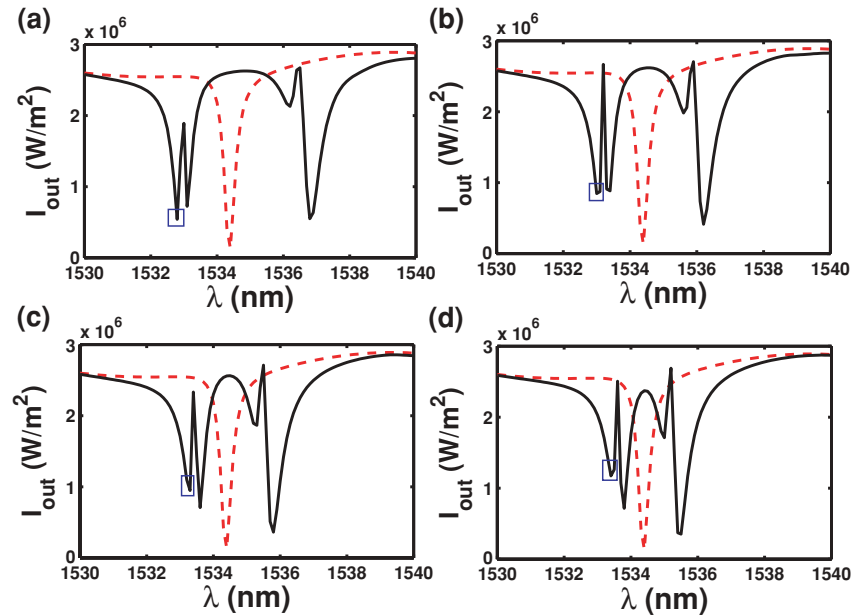

FIG. 3. (Color online) Resonance of the single-ring resonator (dashed line) and double-ring resonator system (solid line) as a function of wavelength for $d$ values of (a) 25, (b) 50, (c) 75, and (d) $100 \mathrm{~nm}$ at $0 \mathrm{~V}$. 
and ring-ring coupling. We focus on controlling the linewidth in addition to the resonance splitting in this way.

Four peaks arise because of the simultaneous presence of direct proximity coupling together with the additional bus-waveguide-mediated coupling between the rings [21]. In other systems such as ballistic electron guides, two-peak splitting occurs since the resonators are coupled only through the bus waveguide. The asymmetry of the line shapes in Fig. 3 is also a characteristic signature of quantum interference. The interference is of Fano type because of the spectrally overlapping subradiant and superradiant decay channels. When the gap between two ring resonators is widened, the proximity coupling between the rings is reduced while the bus-ring coupling remains unchanged. The splitting about the isolated ring resonance between the symmetrically placed symmetric and antisymmetric modes decreases. The further splitting of these modes due to the ring-bus interaction is independent of $d$ but their width ( $\Gamma$, full width at half maximum) varies with it.

In addition to the splitting into subradiant and superradiant channels, the linewidths have oscillatory behavior with $d$. This signature of the Dicke effect is due to the spatial interference of radiation from decaying quasibound states of the rings coupled to the waveguide at separated locations inherent to the collective nature of the system. In our case, Dicke oscillations of symmetric and antisymmetric modes are translated into further split modes. The effect can be understood as analogous to the level repulsion between coherently coupled degenerate bound states [14]. Interacting (interfering) decay channels also split, along with the interaction caused by splitting of the resonances. If the linewidths are represented as imaginary parts of the resonances, the symmetric and antisymmetric modes lie $180^{\circ}$ out of phase in the complex plane. The interaction channel through the bus waveguide introduces $d$-dependent phase accumulation to the mode freely propagating between the rings along the waveguide. This is translated as a sinusoidal coupling between the rings or a rotation operation in the complex energy plane. The resonances collapse back onto the isolated ring value in a spiraling fashion when the gap between the rings is increased. During this spiral motion, the imaginary parts periodically become vanishing and finite, and hence oscillation occurs.

When two identical systems interact via proximity coupling then perturbation theory yields a level repulsion for a resonance, that is, $E_{0}$, in the form $E_{ \pm}=E_{0} \pm \delta$, where $\delta$ denotes the level shift, whose strength depends on the interaction. The corresponding states are the symmetric and antisymmetric modes. Here, \pm is interpreted as the phase difference; it can be expressed as $\exp (i \pi)$ and can be viewed as the two modes (more strictly speaking the energies of the modes) lying opposite $\left(180^{\circ}\right.$ out of phase) to each other relative to the midpoint $E_{0}$. These energies are mapped to the complex plane for the following reason: When there are decay channels present, the system becomes an open one that can be described by non-Hermitian Hamiltonians. Recently, this approach was systematically used to interpret superradiant phase transitions in one-dimensional nanostructures in terms of non-Hermitian effective Hamiltonians [28]. The spectrum of the system consists of complex eigenenergies where the imaginary parts represent the decay rates or the linewidths. If the decay rate
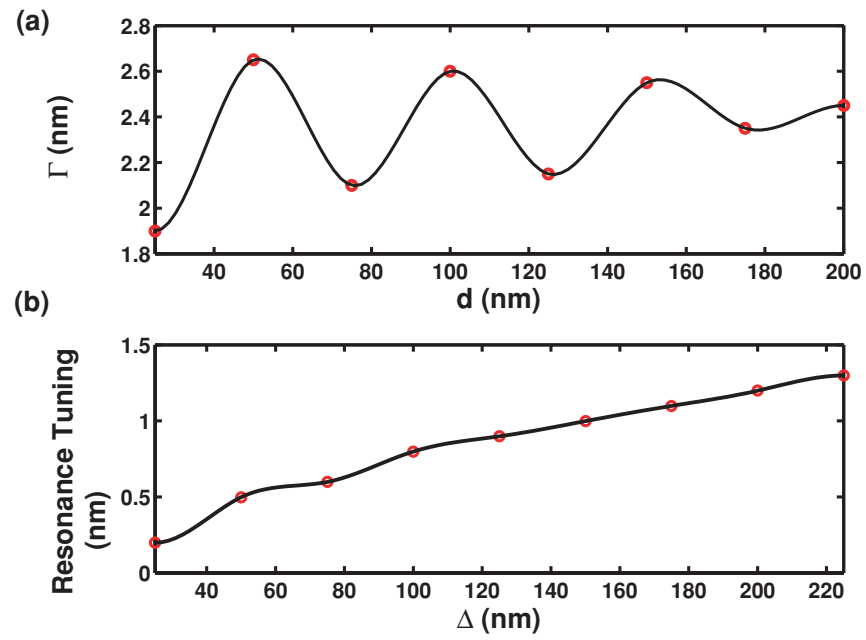

FIG. 4. (Color online) (a) Width of the picked resonance in Fig. 3(a) vs the gap between the two ring resonators. (b) Resonance tuning vs additional gap distance above $d=25 \mathrm{~nm}$ at $0 \mathrm{~V}$.

for a single isolated subsystem is denoted by $\Gamma_{0}$, the level anticrossing in the complex energy plane can be expressed as $E_{ \pm}=E_{0} \pm \delta-i\left(\Gamma_{0} \pm \gamma\right)$. Here, $\gamma$ represents the interference term arising from the interacting decay channels. These complex energies again lie out of phase and are centered about the pole $E_{0}-i \Gamma_{0}$. In the case of the two-ion model of Dicke, $\gamma$ is proportional to the zeroth-order spherical Bessel function of $k d$ with $k$ and $d$ being the photon wave number and the distance between the ions, respectively [11]. It has the same form of distance dependence in the case of coupled quantum dots [11], while for the ballistic electron waveguide with two ripple cavities, it becomes a sinusoidal function [14]. Our numerical results reveal that the linewidths for the system of double-ring cavities side-coupled to a waveguide behave like the original Dicke model of two ions.

Choosing the most energetic resonance, labeled with a box in Fig. 3(a), at $d=25 \mathrm{~nm}$, we investigate the dependence of the resonance width and resonance wavelength on the spatial separation between the rings. Figure 4(a) shows the variation in the width of the resonance as a function of the gap between the two ring resonators. The coupling between the rings decreases with increasing distance and the oscillations eventually disappear. The width saturates at the single-ring value at long distances. In the one-dimensional system of a two-ripple ballistic electron waveguide, sinusoidal periodic behavior of the resonance width is found because the coupling does not decay with distance [14]. Our situation is similar to the traditional Dicke systems in higher dimensions, where the coupling decreases with increasing distance [11,12]. Resonance tuning as a function of the gap amount, $\Delta$, additional to $d$ is shown in Fig. 4(b). As the coupling decreases with distance, the splitting of the collective modes decreases. Thus, the most energetic mode becomes less and less different from the single-ring resonance.

In the double-ring resonator system, we expect an enhancement of the rate of transmission only in the case of the superradiant channel and a decrease in the case of the subradiant channel. The overall intensity will be the same as at the input (or most likely less in practice because of 
emission into free space from the rings or other losses). The long-lived antisymmetric quasibound state of the double-ring system (in Dicke's term, the superradiant state) decays twice as fast as the quasibound state of the single-ring system. The short-lived symmetric quasibound state of the double-ring system (in Dicke's term, the subradiant state) exists for twice as long as the quasibound state of the single-ring system. Our numerical results indicate that the superradiant decay is about three times faster than the decay of a single isolated ring. For a two-atom system, the enhancement in the linewidth is 2.5 times more than for the single-atom system [11]. In the double-ring system, the linewidth of a single peak or two adjacent resonances increases while that of the other single peak or two adjacent resonances decreases.

In addition to the linewidth changes, superradiant scattering and quadratic enhancement of the scattered intensity with the number of emitters can also be found in our system. To show that, another waveguide can be introduced above the doublering system to serve as the output or scattering channel. Then the input channel can be viewed as the drive or incoming field, which is the TE-polarized Gaussian beam sent from the input port at the left side of the lower waveguide. The transmission characteristics of the system are determined by analysis of the beam detected in the output port at the left side of the upper waveguide. We find that the output intensity increases quadratically. This result shows that our system is capable of exhibiting all the signatures of the Dicke effect. More detailed analysis of the two-waveguide system coupled with more ring resonators should be the subject of further studies.

\section{DOUBLE-RING RESONATOR SYSTEM UNDER APPLIED VOLTAGE}

We examine possible active control of the signatures of the Dicke effect demonstrated in Fig. 4 by considering another energetic resonance found at a larger separation between the rings and labeled with a box in Fig. 3(d) at $d=100 \mathrm{~nm}$. When the potential applied from the electrodes is turned on, the linewidth and the resonance are changed as shown in Fig. 5.
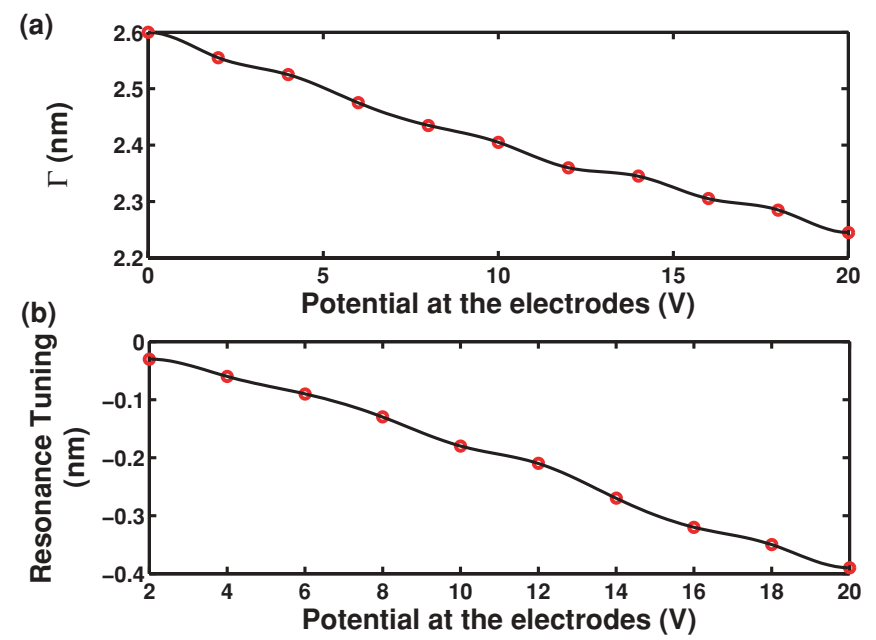

FIG. 5. (Color online) (a) Resonance width and (b) resonance tuning of the picked resonance in Fig. 3(d) at $d=100 \mathrm{~nm}$ vs applied voltage from the electrodes.
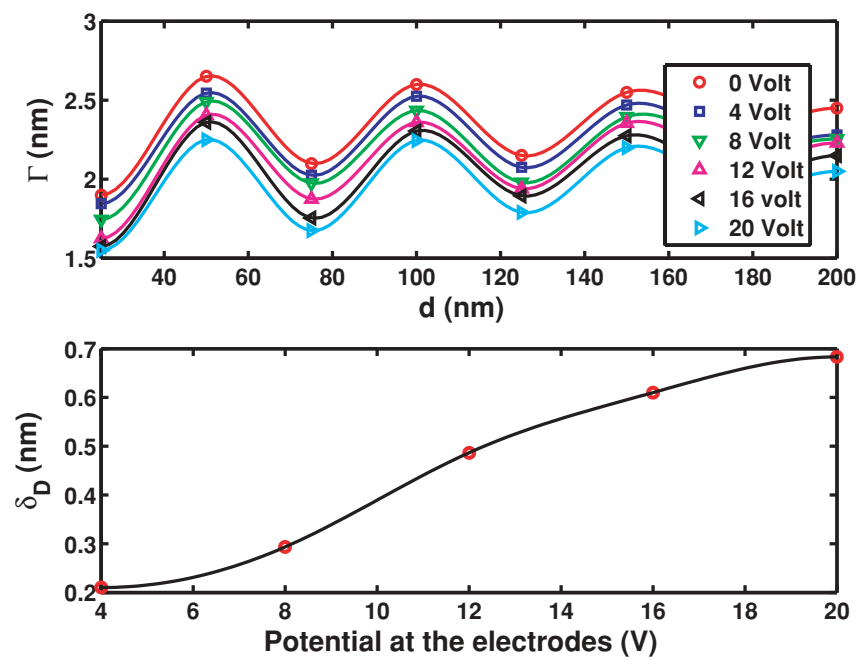

FIG. 6. (Color online) (a) Width of the chosen resonance in Fig. 3(a) vs the gap between two ring resonators for different voltage values applied from the electrodes. (b) Increase in the periodicity of the resonance width function for different voltage values.

The linewidth decreases with increasing potential, which aligns the directors such that the cladding index increases to $n_{e}$ in the coupler zones. This locally reduces the index contrast with the silicon guides and enhances the field penetration. Hence, the proximity coupling between the resonators increases. The chosen most energetic mode becomes further split from the single-ring resonance with the increasing coupling coefficient. The applied voltage and the spatial separation between the rings have opposite effects on the coupling resonators.

We analyze the influence of the external potential on the periodicity of $\Gamma$ as a function of $d$. Figure 6(a) is in agreement with the earlier observations in Fig. 5(a) that the resonance width decreases with increasing applied voltage. The periodicity of $\Gamma$ when no voltage is applied [see Fig. 3(a)] is determined to be $\lambda_{D}=48.92 \mathrm{~nm} ; \delta_{D}$ is the change of the period from this value when the potential is applied. Figure $6(\mathrm{~b})$ shows that the periodicity of $\Gamma$ increases steadily with increase in the potential applied from the electrodes. Enhanced coupling of the rings increases the splitting of symmetric and antisymmetric modes. This brings the chosen most energetic resonance closer to the waveguide mode. Thus, the free propagation and the associated phase accumulation between the rings occur at smaller frequency or at larger period. In the complex energy plane, the rate of rotation with $d$ is reduced.

\section{NUMERICAL METHOD}

We do the numerical analysis using the FEM. We analyze the system in two steps. First, we calculate the change in the refractive index of the NLC cladding with applied potential from the electrodes by solving an electrostatics problem. Second, the new refractive index value is used for the NLC cladding environment in a wave equation to analyze fully the resonance nature of the microring resonator system under applied voltage. 
The electrostatics problem in the whole system including the silicon rings and waveguide and the NLC cladding is given by

$$
\nabla \cdot \varepsilon_{0} n^{2} \nabla V=0
$$

where $n$ is the refractive index of each material (for the NLC, we use $n_{e}=1.744$ and $n_{o}=1.517$, and for silicon, $n=3.48$ ). We apply electric shielding boundary conditions in the boundaries around the overall system except for the electrodes:

$$
\mathbf{n} \cdot \mathbf{D}=-\nabla_{t} \cdot \varepsilon_{0} \nabla_{t} V,
$$

where $\mathbf{n}$ is the unit vector in the direction normal to the plane of the resonators. At the electrodes, a potential $V_{0}$ is applied from the left side and the right side is grounded.

After the electrostatics problem has been solved, we have the following wave equation for the NLC medium:

$$
\nabla \times\left(\nabla \times E_{z} \hat{z}\right)-n^{2} k_{0}^{2} E_{z} \hat{z}=\mathbf{0},
$$

where $n$ is the refractive index of the NLC cladding defined in Eq. (1) calculated by solution of the electrostatics problem and fed into the wave equation to be used as the new value of the refractive index of the NLC cladding. In the silicon rings and waveguide, the wave equation

$$
\nabla \times\left(\nabla \times E_{z} \hat{z}\right)-\left(n^{2}-\frac{j \sigma}{\omega \varepsilon_{0}}\right) k_{0}^{2} E_{z} \hat{z}=\mathbf{0}
$$

is used, where $n=3.48$ and $\sigma=10^{-12}(\mathrm{~S} / \mathrm{m})$ (silicon). In the boundaries around the overall system except at the ports, an impedance boundary condition is applied:

$$
\mathbf{n} \times \mathbf{H}+\sqrt{n^{2}-\frac{j \sigma}{\omega}} \mathbf{n} \times(\mathbf{E} \times \mathbf{n})=\mathbf{0} .
$$

At the ports, a Gaussian-shape beam is introduced as shown:

$$
E_{z}=E_{0} \exp \left(\frac{\left(-y-y_{0}\right)^{2}}{r^{2}}\right),
$$

where $y_{0}$ is the center of the waveguide and $r$ is $300 \mathrm{~nm}$.

\section{CONCLUSION}

We have examined the resonances and their widths in an all-optical system of a pair of ring resonators side-coupled to an optical waveguide in a NLC environment. We found that the system exhibits all the key signatures of the Dicke effect: splitting of the lifetimes into slow- and fast-decaying channels in an oscillatory manner with the separation of the resonators. The energies and the lifetimes of the quasibound states of the resonators can be controlled with an applied voltage which allows for tunable longer range interactions between the resonators. Such reversible active control can be used to examine coherent collective effects, decoherence, and superradiant phase transitions. In addition, fine tuning of the finesse and full spectral range can be exploited for multiring systems, such as CROW [29] and SCISSOR [30] configurations, as well as rings coupled to multiple waveguides. These systems have many applications, particularly in optical logic and memory, filtering, reflectivity, and optical switching.

\section{ACKNOWLEDGMENTS}

The authors acknowledge useful comments by A. Serpenguzel, H. Altug, H. Tureci, and A. Mertiri.
[1] R. H. Dicke, Phys. Rev. 89, 472 (1953).

[2] R. H. Dicke, Phys. Rev. 93, 99 (1954).

[3] M. Hubner, J. Kuhl, T. Stroucken, A. Knorr, S. W. Koch, R. Hey, and K. Ploog, Phys. Rev. Lett. 76, 4199 (1996).

[4] A. Christ, Y. Ekinci, H. H. Solak, N. A. Gippius, S. G. Tikhodeev, and O. J. F. Martin, Phys. Rev. B 76, 201405(R) (2007).

[5] T. Brandes, Phys. Rep. 408, 315 (2005).

[6] V. M. Apel, P. A. Orellana, and M. Pacheco, Nanotechnology 19, 355202 (2008).

[7] P. A. Orellana, F. Dominguez, and E. Diez, Physica E 35, 126 (2006).

[8] P. A. Orellana, M. L. Ladron de Guevara, and F. Claro, Phys. Rev. B 70, 233315 (2004).

[9] E. V. Podivilov and D. A. Shapiro, JETP Lett. 56, 449 (1992).

[10] T. Vorrath and T. Brandes, Phys. Rev. B 68, 035309 (2003).

[11] T. Brandes and B. Kramer, Phys. Rev. Lett. 83, 3021 (1999).

[12] T. V. Shahbazyan and M. E. Raikh, Phys. Rev. B 49, 17123 (1994).

[13] H. Lee and L. E. Reichl, Phys. Rev. B 77, 205318 (2008).
[14] H. Lee and L. E. Reichl, Phys. Rev. B 79, 193305 (2009).

[15] F. C. Blom, D. R. van Dijk, H. J. W. M. Hoekstra, A. Driessen, and T. J. A. Popma, Appl. Phys. Lett. 71, 747 (1997).

[16] M. T. Hill, H. J. S. Dorren, T. de Vries, X. J. M. Leitjens, J. H. den Besten, B. Smalbrugge, Y.-S. Oei, H. Binsma, G.-D. Khoe, and M. K. Smit, Nature (London) 432, 206 (2004).

[17] P. Anderson, Phys. Rev. 124, 41 (1961).

[18] U. Fano, Phys. Rev. 124, 1866 (1961).

[19] B. Maune, R. Lawson, C. Gunn, A. Scherer, and L. Dalton, Appl. Phys. Lett. 83, 4689 (2003).

[20] I. Chremmos and N. Uzunoglu, IEEE Photonics Technol. Lett. 17, 2110 (2005).

[21] Y. Chung, D.-G. Kim, and N. Dagli, J. Lightwave Technol. 24, 1865 (2006).

[22] B. E. A. Saleh and M. C. Teich, Fundamentals of Photonics (Wiley, New York, 1991).

[23] D. Dunmar and K. Toniyama, Handbook of Liquid Crystals (Wiley-VCH, New York, 1998), Vol. 1, p. 215.

[24] P. J. Collings, Liquid Crystals: Nature's Delicate Phase of Matter (Princeton University Press, Princeton, NJ, 2002).

[25] J. Poon, J. Scheuer, and A. Yariv, IEEE Photonics Technol. Lett. 16, 1331 (2004). 
[26] J. Poon, J. Scheuer, S. Mookherjea, G. T. Paloczi, Y. Huang, and A. Yariv, Opt. Express 12, 90 (2004).

[27] For our numerical work, we have mainly used the computer codes MATHEMATICA (Wolfram Research), MATLAB (The MathWorks), and COMSOL MULTIPHYSICS (Comsol).
[28] G. L. Celardo and L. Kaplan, Phys. Rev. B 79, 155108 (2009). [29] A. Yariv, Y. Xu, R. K. Lee, and A. Scherer, Opt. Lett. 24, 711 (1999).

[30] J. E. Heeber, R. W. Boyd, and Q.-H. Park, J. Opt. Soc. Am. B 19, 722 (2002). 